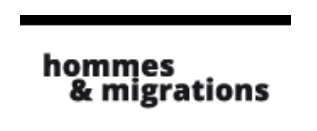

Hommes \& migrations

Revue française de référence sur les dynamiques

migratoires

$1311 \mid 2015$

Femmes et migrations

\title{
Un regard statistique sur la visibilité des migrantes dans l'espace public en Espagne
}

Participation associative et participation aux élections municipales

\section{Celio Sierra-Paycha}

\section{(2) OpenEdition}

Journals

Édition électronique

URL : http://journals.openedition.org/hommesmigrations/3288

DOI : $10.4000 /$ hommesmigrations.3288

ISSN : 2262-3353

Éditeur

Musée national de l'histoire de l'immigration

Édition imprimée

Date de publication : 1 juillet 2015

Pagination : 93-102

ISBN : 978-2-919040-32-2

ISSN : 1142-852X

Référence électronique

Celio Sierra-Paycha, « Un regard statistique sur la visibilité des migrantes dans l'espace public en Espagne », Hommes \& migrations [En ligne], 1311 | 2015, mis en ligne le 01 juillet 2018, consulté le 30 avril 2019. URL : http://journals.openedition.org/hommesmigrations/3288 ; DOI : 10.4000/ hommesmigrations.3288 


\title{
UN REGARD STATISTIQUE SUR LES MIGRANTES EN ESPAGNE \\ PARTICIPATION ASSOCIATIVE ET AUX ÉLECTIONS MUNICIPALES
}

par CELIO SIERRA-PAYCHA, doctorant à l'université de Poitiers, Migrinter (UMR 7301), et à l'Ined, (Unité 6 Mobilité, Logement, Entourage).

\author{
L'enquête nationale sur les immigrants en Espagne a permis \\ de collecter des données sur la participation associative \\ et le vote aux élections municipales des femmes migrantes. \\ Portant sur 8500 femmes nées à l'étranger et résidant \\ en Espagne depuis plus d'un an, elle offre l'occasion d'analyser \\ en profondeur les composantes de leur visibilité dans l'espace \\ public espagnol. La diversité de ses modalités, de la pratique \\ d'un sport en club à la participation à une organisation politique, \\ reflète en partie celle de leurs situations migratoires.
}

L'enquête nationale sur les immigrants ${ }^{1}$ a été réalisée en 2007 dans un contexte d'accroissement sans précédent de la population migrante en Espagne. Elle permet de pallier l'absence de données sur ce contingent de migrants arrivés essentiellement depuis le début des années 2000, en plein boom immobilier espagnol ${ }^{2}$. Les études sur les migrations en Espagne n'ont pas manqué de souligner la "nouveauté, la magnitude et la rapidité" du changement ${ }^{3}$. En quelques années, l'Espagne est passée d'un pays d'émigration à une terre d'immigration.
Malgré tous les mouvements migratoires qui l'ont affectée ${ }^{4}$ depuis la date de cette enquête (retours, réémigrations, nouvelles immigrations), cette enquête reste sans équivalent en Europe grâce à la taille de son échantillon ( $N=14$ 465) et à son questionnaire détaillé. Ceux-ci permettent d'explorer en profondeur le processus migratoire en Espagne à la veille de la grande récession de 2008-2010. L'institut national de stratistiques (ENI) interroge la visibilité des migrants dans la société d'accueil au travers de la participation des enquêtés à deux types d'as- 
sociations ou d'organisations. D'une part, l'enquête capte la participation à des associations ou à des organisations spécifiquement tournées vers les migrants, au sein desquelles on compte les aides aux migrants, les organisations sportives, les associations culturelles ou éducatives, les associations religieuses, les autres associations à vocation sociale : "Participez-vous à l'un de ces groupes ou associations spécifiquement destinés aux étrangers : organisations d'aide aux migrants, associations et clubs sportifs, groupes à vocation éducative ou culturelle, entités et groupes religieux, autres groupes à caractère social ${ }^{5}$ ?" D'autre part, elle interroge la participation à des organisations qui ne sont pas spécifiquement tournées vers les migrants - nous les appellerons ici des "associations généralistes" -, parmi lesquelles on compte les organisations non gouvernementales (ONG), les partis politiques ou syndicats, les organisations religieuses, les associations culturelles ou éducatives, les associations sportives et les autres associations à vocation sociale: "Participez-vous à l'un de ces groupes ou associations non spécifiquement destinés aux étrangers ${ }^{6}$ ?" Enfin, une question permet de considérer les femmes migrantes ayant voté au moins une fois à des élections municipales en Espagne. Précisons qu'avant 2008, “hormis les citoyens européens, seuls les Norvégiens avaient le droit de vote en Espagne ${ }^{7}$.

\section{La diversité des champs migratoires en Espagne}

La diversité des profils de migrantes installées en Espagne est manifeste, tant entre les différents groupes nationaux qu'au sein des champs migratoires eux-mêmes (tableaux 1 et 2).
Dans l'ensemble, elles sont en majorité lettrées et diplômées : plus de la moitié a au moins un diplôme équivalent au baccalauréat français et $95 \%$ d’entre elles savent lire et écrire.

On peut identifier quatre grandes zones d'émigration vers l'Espagne avant 2007 : l'Europe de l'Ouest, l'Europe de l'Est, l'Amérique latine et l'Afrique du Nord. Si la plupart déclarent l'espagnol comme langue maternelle, parmi les non-hispanophones d'origine, rares sont les femmes qui ne parlent pas du tout la langue de Cervantès (seules $6 \%$ d'entre elles). Leur niveau d'espagnol est en général plutôt bon $(13 \%)$ voire très bon $(18 \%)^{8}$. Cependant, la maîtrise de l'espagnol varie fortement selon les langues maternelles : $56 \%$ des Portugaises le parlent très bien contre seulement $16 \%$ des Ukrainiennes. Une majorité de ces femmes sont actives : $55 \%$ travaillent au moment de l'enquête et seules $14 \%$ sont en recherche d'emploi, $9 \%$ sont étudiantes et $8 \%$ sont à la retraite. Là encore, les variations sont fortes selon les groupes. Les Boliviennes ou les Équatoriennes diffèrent par leur activité salariée des Marocaines ou des Britanniques qui demeurent plutôt femmes au foyer ${ }^{9}$. Ces dernières sont, par ailleurs, davantage à la retraite, tout comme les Cubaines, tandis que les Mexicaines et les Russes sont plus souvent étudiantes que les autres migrantes. De nombreuses femmes pratiquent d'ailleurs la pluriactivité, combinant un emploi officiel et les travaux domestiques. À ce titre, 53 $\%$ des migrantes déclarent être femmes au foyer (tableau 2).

Les activités professionnelles des femmes migrantes ne correspondent pas systématiquement à leur diplôme. En effet, les deux groupes socioprofessionnels dominants sont les travailleuses non qualifiées et les employées dans le sec-

4. Colectivo IOÉ, "Impactos de la crisis sobre la población inmigrante”, Organización Internacional para las Migraciones, document de travail, Ministerio de Asuntos Sociales, Madrid, 2012 ; Celio Sierra-Paycha, «La dinámica espacial y demográica de un sistema migratorio en contexto de crisis: el caso de los colombianos en España a partir de la estadística de variaciones residenciales (2005-2012)", in Actes du colloque XIV Congreso de Población-Asociación de Geógrafos Españoles, Séville, 2014. 5. "¿Participa en algunos de los siguientes grupos o asociaciones orientados especificamente a extranjeros ? Organizaciones de ayuda al inmigrante/ Asociaciones y clubs deportivos/ Grupos educativos y cuturales/ Entidades y grupos religiosos/ Otros grupos de caracter social ?”, Instituto Nacional de Estadísticas (Espagne), op. cit. 6. “'Participa en algunos de los siguientes grupos o asociaciones orientados no especificamente a extranjeros?", Ibid. 7. Hervé Andres, "Réciprocité et droit de vote des étrangers en Espagne et au Portugal”, in P@ges Europe, Paris, La Documentation française, 2013. 8. Il s'agit d'un jugement émanant des migrantes elles-mêmes. 9. La question posée est : "Vous consacrez-vous aux travaux domestiques ?" 
teur des services; seules $4 \%$ sont cadres et $9 \%$ exercent des professions intellectuelles et scientifiques. Cette asymétrie entre diplôme et profession est caractéristique des pays occidentaux à forte immigration ${ }^{10}$. Dans un contexte d'augmentation du travail féminin et de retrait des femmes natives de la sphère domestique, les métiers du care constitueraient une niche professionnelle pour les migrantes. La situation des Ukrainiennes est la plus paradoxale : $43 \%$ ont réalisé des études supérieures (soit le groupe le plus qualifié avec les Mexicaines), mais plus de la moitié occupent des emplois de travailleuses non qualifiées. Il ne s'agit pas, pour autant, de généraliser la tendance à la migration qualifiée en Espagne. Par exemple, 41 \% des Marocaines et 55 \% des Portugaises n'ont aucun diplôme ou ont effectué seulement des études primaires ; $30 \%$ des premières et $15 \%$ des secondes ne savent ni lire ni écrire.

Au plan démographique, la population migrante en Espagne apparaît relativement jeune (27 \% ont entre 16 et 29 ans et $44 \%$ ont entre 30 et 44 ans). Une majorité a des enfants (71\%) et plus de la moitié est mariée, contre un tiers demeurant célibataire ${ }^{11}$. Les célibataires sont particulièrement surreprésentées parmi les groupes andins (Équateur, Colombie, Bolivie). Si, pour de nombreux groupes nationaux, les premières migrations vers l'Espagne remontent aux années 1990, force est de constater qu'une migrante sur cinq est arrivée avant 1990. Les Françaises forment d'ailleurs le groupe le plus anciennement installé sur le territoire espagnol (75 \% sont arrivées avant 1990).

La nationalité espagnole est inégalement distribuée parmi les groupes nationaux. Alors que les Suisses, les Françaises, les Cubaines et les Mexicaines ont très souvent la nationalité espagnole, ce n'est pas le cas des Boliviennes ou des femmes d'Europe de l'Est - quasiment aucune ne l'a.
Ces différences sont le produit de l'histoire migratoire de l'Espagne, de nombreux descendants d'émigrés espagnols étant "retournés" en Espagne après la transition démocratique des années 1980. Bien quétant nés dans un autre pays, ils ont des origines espagnoles et peuvent bénéficier à ce titre de la nationalité ${ }^{12}$. D'ailleurs, $69 \%$ des Suisses et 53 \% des Françaises résidant en Espagne en 2007 déclarent l'espagnol comme langue maternelle, ce qui montre le poids des descendants d'émigrés espagnols dans les flux migratoires depuis ces deux pays vers l'Espagne.

Si certains groupes nationaux correspondent à des figures particulières de migrants - les retraitées britanniques, les jeunes Boliviennes dans le secteur des services à la personne, les cadres allemands, les étudiantes mexicaines, les Ukrainiennes surqualifiées travaillant comme employées domestiques -, la diversité des situations au sein des champs migratoires eux-mêmes On peut identifier quatre grandes zones d'émigration vers l'Espagne avant 2007: l'Europe de l'Ouest, l'Europe de l'Est, l'Amérique latine et l'Afrique du Nord. est frappante. Le cas des Colombiennes en témoigne: si les données statistiques confirment une majorité de travailleuses non qualifiées, de récents travaux ont montré l'existence d'autres profils plus qualifiés ${ }^{13}$.

\section{Une visibilité différente selon le genre}

La visibilité dans l'espace public est entendue ici au sens d'une participation officialisée d'un individu à une association, organisation ou groupe - en dehors du cadre professionnel - et du vote lors d'élections municipales dans la société d'accueil. Sans disposer d'éléments de comparaison avec la population espagnole à la même époque permettant de nuancer 
/// Tableau $11^{14}$ : Caractéristiques démographiques

et projets migratoires des femmes migrantes en Espagne

selon le pays de naissance.

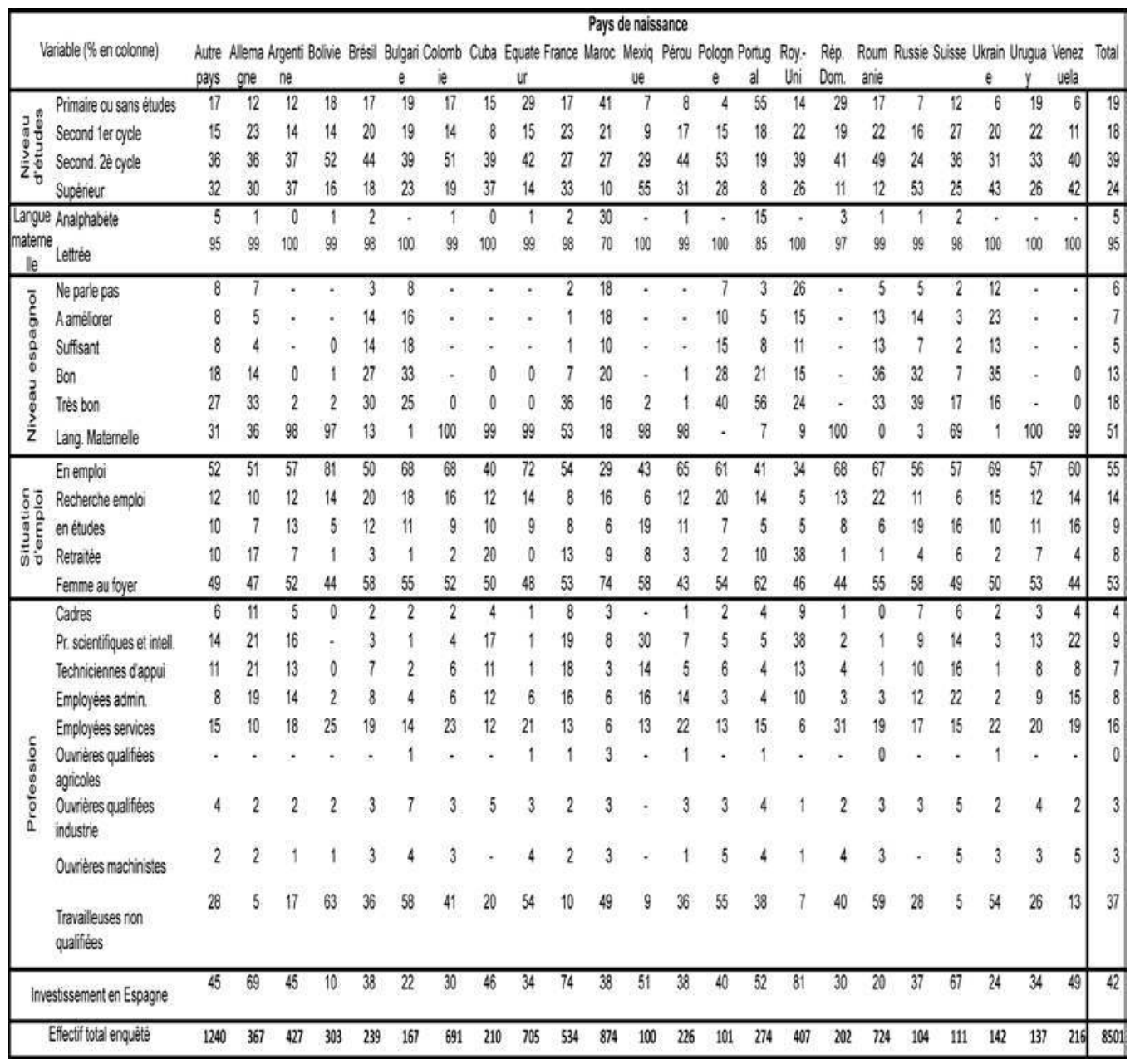


/// Tableau 2 : Caractéristiques sociales des femmes migrantes

en Espagne selon le pays de naissance.

Pays de naissance

Variable (\% encolonne) Autre Allema Argent Bolive Bresil Bugari Colomb Cuba Equate France Maroc Mexiq Perou Pologn Portug Roy. Rép. Roum Russie Suisse Ukrain Urugua Venez Total

\begin{tabular}{|c|c|c|c|c|c|c|c|c|c|c|c|c|c|c|c|c|c|c|c|c|c|c|c|c|c|c|}
\hline & & pars & gne & ne & & & $e$ & ie & & Uf & & & Le & & e & al & Uni & Dom & anie & & & & e & $y$ & uela & \\
\hline \multirow{4}{*}{ 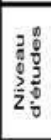 } & Primaire ou sans études & 17 & 12 & 12 & 18 & 17 & 19 & 17 & 15 & 29 & 17 & 41 & 7 & 8 & 4 & 55 & 14 & 2 & 17 & & 7 & 12 & 6 & 19 & 6 & 19 \\
\hline & Second 1er cycle & 15 & 23 & 14 & 14 & 20 & 19 & 14 & 8 & 15 & 23 & 21 & 9 & 17 & 15 & 18 & 22 & 1 & 22 & & 16 & 27 & 20 & 22 & 11 & 18 \\
\hline & Second ze oyce & 36 & 36 & 37 & 52 & 44 & 39 & 51 & 39 & 42 & 27 & 27 & 29 & 44 & 53 & 19 & 39 & 4 & 49 & & 24 & 36 & 31 & 33 & 40 & 39 \\
\hline & Superieur & 32 & 30 & 37 & 16 & 18 & 23 & 19 & 37 & 14 & 33 & 10 & 55 & 31 & 28 & 8 & 26 & 1 & 12 & & 53 & 25 & 43 & 26 & 42 & 24 \\
\hline \multirow{2}{*}{\multicolumn{2}{|c|}{$\begin{array}{l}\text { Langue Analphabäte } \\
\text { materne Lettré } \\
\text { le Letee }\end{array}$}} & 5 & 1 & 0 & 1 & 2 & $\cdot$ & 1 & 0 & 1 & 2 & 30 & $\cdot$ & 1 & ? & 15 & 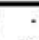 & & 1 & & $\overline{1}$ & 2 & 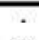 & 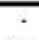 & . & 5 \\
\hline & & 95 & 99 & 100 & 99 & 98 & 100 & 99 & 100 & 99 & 98 & 70 & 100 & 99 & 100 & 86 & 100 & 9 & 96 & & 99 & 98 & 100 & 100 & 100 & 95 \\
\hline \multirow{6}{*}{ 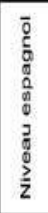 } & Ne parle pas & 8 & 7 & - & - & 3 & 8 & . & $\cdot$ & $\cdot$ & 2 & 18 & . & . & 7 & 3 & 26 & 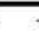 & 5 & & 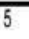 & 2 & 12 & $\cdot$ & & 6 \\
\hline & Aaméliorer & 8 & 5 & . & . & 14 & 16 & . & . & . & 1 & 18 & . & . & 10 & 5 & 15 & . & 13 & & 14 & 3 & 23 & - & . & 7 \\
\hline & Suffisant & 8 & 4 & . & 0 & 14 & 18 & . & . & . & 1 & 10 & . & . & 15 & 8 & 11 & . & 13 & & 7 & 2 & 13 & . & . & 5 \\
\hline & Bon & 18 & 14 & 0 & 1 & 27 & 33 & . & 0 & 0 & 7 & 20 & . & 1 & 28 & 21 & 15 & . & 30 & & 32 & 7 & 35 & . & 0 & 13 \\
\hline & Très bon & 27 & 33 & 2 & 2 & 30 & 25 & 0 & 0 & 0 & 36 & 16 & 2 & 1 & 40 & 56 & 24 & . & 3 & & 39 & 17 & 16 & . & 0 & 18 \\
\hline & Lang Naternele & 31 & 36 & 98 & 97 & 13 & 1 & 100 & 99 & 99 & 53 & 18 & 98 & 98 & . & 7 & 9 & 10 & c & & 3 & 69 & 1 & 100 & 99 & 51 \\
\hline \multirow{5}{*}{ 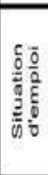 } & En emploi & 52 & 51 & 57 & 81 & 50 & 68 & 68 & 40 & 72 & 54 & 29 & 43 & 65 & 61 & 41 & 34 & 6 & 6 & & 56 & 57 & 69 & 57 & 60 & 55 \\
\hline & Recherche emploi & 12 & 10 & 12 & 14 & 20 & 18 & 16 & 12 & 14 & 8 & 16 & 6 & 12 & 20 & 14 & 5 & 1 & 22 & & 11 & 6 & 15 & 12 & 14 & 14 \\
\hline & en etudes & 10 & 7 & 13 & 5 & 12 & 11 & 9 & 10 & 9 & 8 & 6 & 19 & 11 & 7 & 5 & 5 & & 6 & & 19 & 16 & 10 & 11 & 16 & 9 \\
\hline & Retraites & 10 & 17 & 7 & 1 & 3 & 1 & 2 & 20 & 0 & 13 & 9 & 8 & 3 & 2 & 10 & 38 & & 1 & & 4 & 6 & 2 & 7 & 4 & 8 \\
\hline & Fermme au foyer & 49 & 47 & 52 & 44 & 58 & 55 & 52 & 50 & 48 & 53 & 74 & 58 & 43 & 54 & 62 & 46 & 4 & 5: & & 58 & 49 & 50 & 53 & 44 & 53 \\
\hline \multirow{9}{*}{$\begin{array}{l}\frac{6}{0} \\
\frac{0}{d} \\
\frac{0}{0} \\
\frac{0}{2}\end{array}$} & Cadres & $\overline{6}$ & 11 & $\overline{5}$ & 0 & 2 & 2 & 2 & 4 & 1 & 8 & 3 & ? & 1 & 2 & 7 & 9 & & 7 & & 7 & $\overline{6}$ & 2 & $\overline{3}$ & 4 & 4 \\
\hline & Pr. scentfiques et intell. & 14 & 21 & 16 & . & 3 & 1 & 4 & 17 & 1 & 19 & 8 & 30 & 7 & 5 & 5 & 38 & & 1 & & 9 & 14 & 3 & 13 & 22 & 9 \\
\hline & Technicennes d'appui & 11 & 21 & 13 & 0 & 7 & 2 & 6 & 11 & 1 & 18 & 3 & 14 & 5 & 6 & 4 & 13 & & 1 & & 10 & 16 & 1 & 8 & 8 & 7 \\
\hline & Employees admin. & 8 & 19 & 14 & 2 & 8 & 4 & 6 & 12 & 6 & 16 & 6 & 16 & 14 & 3 & 4 & 10 & & 3 & & 12 & 22 & 2 & 9 & 15 & 8 \\
\hline & Employ & 15 & 10 & 18 & 25 & 19 & 14 & 23 & 12 & 21 & 13 & 6 & 13 & 22 & 13 & 15 & 6 & 3 & 18 & & 17 & 15 & 22 & 20 & 19 & 16 \\
\hline & $\begin{array}{l}\text { Ouvrières qualifiées } \\
\text { agriodes }\end{array}$ & . & . & . & . & . & 1 & . & . & 1 & 1 & 3 & . & 1 & . & 1 & . & & c & & . & . & 1 & . & . & 0 \\
\hline & $\begin{array}{l}\text { Ouvrieres qualifiées } \\
\text { industie }\end{array}$ & 4 & 2 & 2 & 2 & 3 & 7 & 3 & 5 & 3 & 2 & 3 & . & 3 & 3 & 4 & 1 & & 3 & & 3 & 5 & 2 & 4 & 2 & 3 \\
\hline & Ouvrieres machinistes & 2 & 2 & 1 & 1 & 3 & 4 & 3 & . & 4 & 2 & 3 & . & 1 & 5 & 4 & 1 & & & & - & 5 & 3 & 3 & 5 & 3 \\
\hline & $\begin{array}{l}\text { Travalleuses non } \\
\text { quaffiets }\end{array}$ & 28 & 5 & 17 & 63 & 36 & 58 & 41 & 20 & 54 & 10 & 49 & 9 & 36 & 55 & 38 & 7 & 4 & 5 & 2 & 28 & 5 & 54 & 26 & 13 & 37 \\
\hline \multicolumn{2}{|c|}{ Investissement en Espagne } & 45 & 69 & 45 & 10 & 38 & 22 & 30 & 46 & 34 & 74 & 38 & 51 & 38 & 40 & 52 & 81 & 3 & 2 & 3 & 37 & 67 & 24 & 34 & 49 & 42 \\
\hline & Ellectifitial enquétét & 1240 & 367 & 427 & 303 & 239 & 167 & 691 & 210 & 705 & 534 & 874 & 100 & 226 & 101 & 27 & 40 & & n & & & 11 & 142 & 137 & 216 & 850 \\
\hline
\end{tabular}


les tendances décrites ici, la visibilité des migrants dans l'espace public apparaît comme un phénomène minoritaire. Quel que soit le type d'organisation retenu, la participation associative est inférieure à $15 \%$ : $15 \%$ des femmes participent à une association généraliste, $7 \%$ à une association de migrants (tableau 3). En revanche, un migrant sur
Alors que les premiers sont surreprésentés dans les associations sportives et dans les organisations politiques, les secondes le sont dans les associations religieuses, les organisations à caractère social et les ONG. On ne peut donc réduire la participation des femmes migrantes dans l'espace public à un décalque de celle des hommes. D'ailleurs, aux élections municipales, les migrantes sont légèrement plus visibles (25,2 \%) que les migrants (23,7 \%). Il s'agit plutôt d'un investissement différencié selon les types d'associations et d'une occupation différente de l'espace public : aux hommes appartiendrait plutôt la visibilité physique et politique à proprement parler, tandis qu'aux femmes migrantes reviendrait l'engagement solidaire et spirituel.

\section{Une participation associative distribuée selon le pays d'origine}

Afin de différencier les groupes nationaux en fonction de leur plus ou moins forte participation à l'espace public, nous nous appuyons ici sur une analyse des correspondances multiples croisant le pays de naissance avec la participation à une association de migrants, à une association généraliste ou aux élections municipales (figure $1^{15}$ ). Il s'agit d'une méthode factorielle permettant de décrire les individus selon une série de variables projetées sous la forme d'un nuage sur un plan. Plus les variables sont proches, plus les individus appartenant à l'une des variables ont de chances d'appartenir à l'autre.

Le nuage se lit d'abord en cherchant les points indiquant les différentes formes de participation. Le point "assomigrantes", en haut à gauche, indique une zone du nuage où les individus participent beaucoup à des associations de migrants, par oppo- 


\section{/// Tableau 3 : Participation associative et électorale des femmes migrantes en Espagne selon le pays de naissance.}

\begin{tabular}{|c|c|c|c|c|c|c|c|c|c|c|c|c|c|c|c|c|c|c|c|c|c|c|c|c|c|}
\hline \multirow{2}{*}{\multicolumn{2}{|c|}{ Variable (\% en colonne) }} & \multicolumn{24}{|c|}{ Pays de naissance } \\
\hline & & $\begin{array}{l}\text { Autre } \\
\text { pays }\end{array}$ & $\begin{array}{l}\text { Alema } \\
\text { gne }\end{array}$ & $\begin{array}{l}\text { Argent } \\
\text { ne }\end{array}$ & Bolvie & Bresil & $\begin{array}{c}\text { Bulgari } \\
\text { e }\end{array}$ & Colom & Cuba & $\begin{array}{c}\text { Equate } \\
\text { ur }\end{array}$ & France & Maroc & $\begin{array}{c}\text { Mexiqu } \\
\mathrm{e}\end{array}$ & Pérou & \multirow{2}{*}{$\begin{array}{c}\begin{array}{c}\text { Pologn } \\
\mathrm{e}\end{array} \\
\end{array}$} & \multirow{2}{*}{$\begin{array}{c}\begin{array}{c}\text { Portug } \\
\text { al }\end{array} \\
\end{array}$} & \multirow{2}{*}{$\begin{array}{r}\begin{array}{r}\text { Roy.- } \\
\text { Uni }\end{array} \\
16\end{array}$} & \multirow{2}{*}{$\begin{array}{r}\text { Rép. } \\
\text { Dom. } \\
7\end{array}$} & $\begin{array}{c}\text { Rouma } \\
\text { nie }\end{array}$ & Russie & Suisse & $\begin{array}{c}\text { Ukrain } \\
\text { e }\end{array}$ & $\begin{array}{l}\text { Unugu } \\
\text { ay }\end{array}$ & $\begin{array}{c}\text { Venez } \\
\text { vela }\end{array}$ & Total \\
\hline \multicolumn{2}{|c|}{ Asso migrantes } & 10 & 7 & 7 & 71 & 9 & $9 \quad 5$ & $5 \quad 7$ & 2 & 25 & 53 & 7 & 5 & 5 & & & & & $\begin{array}{l}7 \quad 6 \\
\end{array}$ & 6 & $6 \quad 3$ & 39 & $9 \quad 13$ & 36 & 7 \\
\hline \multirow{5}{*}{ 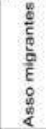 } & Aide migrants & 3 & 1 & 2 & 4 & 4 & 3 & 3 & 1 & 2 & 0 & 2 & 2 & 1 & 2 & 0 & 2 & 3 & 2 & 2 & 1 & 1 & 5 & 2 & 2 \\
\hline & Sport & 1 & 1 & 1 & 2 & 1 & 0 & 0 & 1 & 1 & 1 & 0 & 0 & 0 & 2 & 0 & 5 & 2 & 0 & 1 & 0 & 0 & 1 & 1 & 1 \\
\hline & Educlaulturel & 2 & 1 & 3 & 2 & 2 & 2 & 1 & 1 & 1 & 1 & 2 & 3 & 1 & 4 & 0 & 6 & 1 & 1 & 2 & 1 & 1 & 3 & 3 & 2 \\
\hline & Religion & 2 & 2 & 2 & 2 & 5 & 1 & 1 & 1 & 1 & 1 & 1 & 1 & 2 & 4 & 0 & 2 & 1 & 3 & 0 & 0 & 0 & 1 & d & 2 \\
\hline & Autre social & 3 & 3 & 2 & 4 & 2 & 1 & 2 & 1 & 1 & 1 & 2 & 0 & 2 & 3 & 1 & 6 & 1 & 1 & 3 & 1 & 1 & 6 & 3 & 2 \\
\hline & ogénérales & 18 & 17 & 25 & 8 & 17 & 8 & 16 & 20 & 8 & 23 & 8 & 23 & 17 & 10 & 14 & 25 & 10 & 8 & 9 & 20 & $\overline{0}$ & 26 & 21 & 15 \\
\hline 0 & ONG & 6 & 4 & 10 & 2 & 5 & 1 & 4 & 5 & 2 & 6 & 3 & 7 & 5 & 1 & 4 & 3 & 1 & 2 & 5 & 5 & 5 & 3 & 3 & 4 \\
\hline है & Polifque & 2 & 2 & 2 & 1 & 1 & 2 & 2 & 1 & 1 & 4 & 1 & 3 & 1 & 1 & 1 & 3 & 2 & 1 & 2 & 5 & 5 & 5 & 5 & 2 \\
\hline 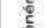 & Religion & 3 & 2 & 4 & 3 & 5 & 1 & 4 & 6 & 2 & 2 & 1 & 5 & 5 & 4 & 3 & 2 & 2 & 2 & 0 & 1 & 1 & 4 & 4 & 3 \\
\hline \& & Édudiculturel & 4 & 5 & 8 & 1 & 3 & 1 & 3 & 5 & 2 & 6 & 2 & 4 & 5 & 3 & 3 & 9 & 2 & 1 & 3 & 6 & 6 & 7 & 7 & 4 \\
\hline 品 & Spot & 5 & 5 & 3 & 1 & 6 & 1 & 2 & 4 & 1 & 8 & 1 & 4 & 3 & 4 & 2 & 7 & 2 & 1 & 1 & 5 & 5 & 8 & 3 & 3 \\
\hline & Autre social & 4 & 4 & 6 & 1 & 5 & 1 & 3 & 6 & 1 & 5 & 2 & 4 & 1 & 2 & 4 & 8 & 2 & 2 & 2 & 3 & 3 & 6 & 4 & 3 \\
\hline Elex & fons munic. & 27 & 56 & 31 & 1 & 20 & 1 & 7 & 37 & 1 & 74 & 26 & 43 & 19 & 8 & 40 & 31 & 21 & 0 & 7 & 70 & 0 & 34 & 50 & 25 \\
\hline Ellect & total enquêté & 1240 & 367 & 427 & 303 & 239 & 167 & 691 & 210 & 705 & 534 & 874 & 100 & 226 & 101 & 274 & 407 & 202 & 724 & 104 & 111 & 142 & 137 & 216 & 8501 \\
\hline
\end{tabular}

Source : Instituto Nacional de Estadísticas (Espagne), op. cit

sition au point "pasmigr" indiquant une zone où l'on participe peu. De même, le point "assogénéral" indique une zone où les individus participent beaucoup aux associations généralistes et s'oppose au point "pasgénéral" marquant la faible participation à ce type d'associations. Enfin, le point "élection municipale" marque une zone où l'on participe beaucoup aux élections municipales et s'oppose au point "pas élection munici" tout à gauche. Le reste des points indique les pays de naissance de façon assez évidente, sauf pour la République dominicaine représentée par "Republi" et le Royaume-Uni représenté par "Reino U”.

Les différents groupes nationaux semblent se répartir selon deux axes diagonaux principaux, un axe opposant la non-participation à la participation en général, et un axe opposant la visibilité plutôt au sein du collectif migrant à la visibilité au sein d'un collectif plus large (les associations généralistes et, plus loin dans l'axe, les élections municipales).

Rappelons que cette méthode fait fi des effets de structure (les positions sociales des individus, l'an- cienneté de la migration en Espagne) jalonnant les différents champs migratoires, mais permet de visualiser les inégalités de fait face à la visibilité associative et électorale des migrantes. Peu importe que les Colombiennes soient arrivées bien plus récemment que les Françaises, que les premières aient bien moins souvent la nationalité espagnole que les secondes, de fait, elles sont moins visibles que les Françaises.

\section{La pluralité des formes d'engagement}

Quelques groupes de migrantes participent beaucoup plus que les autres à l'espace public : les Britanniques, les Uruguayennes, les Argentines, les Mexicaines, les Vénézuéliennes, les Allemandes, les Suisses et les Françaises. Au sein de ce groupe, on distingue d'une part les Françaises, les Suisses et les Allemandes, surreprésentées lors des élections municipales (voir le tableau 3), et, d'autre part, les 
Britanniques et les Uruguayennes surreprésentées, quant à elles, dans les associations de migrants et dans les associations généralistes. D’autres groupes de migrantes sont en revanche plutôt absents de l'espace public: les Bulgares, les Roumaines, les Polonaises, les Russes, les Équatoriennes, les Boliviennes, les Dominicaines, les Colombiennes et les Marocaines. Enfin, un groupe intermédiaire est formé par les Péruviennes, les Brésiliennes, les Cubaines, les Portugaises et les femmes nées dans d'autres pays.

Au-delà de ces inégalités générales de participation, on observe quelques spécificités associatives selon les origines (tableau 3). Les Britanniques pratiquent davantage le sport en club que
D’une manière générale, ce sont les femmes les plus

anciennement installées en Espagne qui se retrouvent

les associations culturelles

et éducatives et parmi

les votantes aux élections municipales. surreprésentées dans les ONG, les autres migrantes, les Uruguayennes sont surreprésentées dans les associations d'aide aux migrants, les Brésiliennes et les Cubaines sont plus souvent engagées dans des organisations religieuses, les Argentines sont considérablement investies dans les ONG, alors que les Françaises et les Suisses sont plus souvent engagées dans des organisations politiques ou syndicales relativement à l'ensemble des migrantes.

L'approche de la migration par le pays de naissance est cependant ardue en raison des effets de structure. On tentera de faire apparaître par la suite quelques inégalités sociales pouvant aller de pair avec une meilleure visibilité dans l'espace public, une fois contrôlé l'effet du pays d'origine ${ }^{16}$.

Pour chaque type de participation, nous avons donc utilisé la méthode des régressions logistiques, en prenant pour référence la non-participation. $\mathrm{Ce}$ type d'outil méthodologique permet de limiter les effets de structure et de raisonner "toutes choses égales par ailleurs", notamment indépendamment de la variable "pays de naissance". Les variables "explicatives" choisies pour l'analyse sont celles des tableaux 1 et 2, auxquelles nous avons ajouté le fait d'appartenir à un champ migratoire féminisé ${ }^{17}$ et la résidence en Espagne. Seuls les effets significatifs au seuil de $10 \%$ sont commentés. À l'exception des associations sportives de migrants et des ONG, les jeunes migrantes ayant moins de 25 ans sont peu visibles dans l'espace associatif espagnol. $\mathrm{Au}$ contraire, on observe une forte visibilité des migrantes les plus âgées, surtout dans les associations d'aide aux migrants. Les jeunes femmes sont par définition invisibles lors des élections municipales parce qu'elles étaient mineures ou à l'étranger lors des dernières élections. Le fait d'avoir des enfants n'a pas d'effet notable sur la participation politique. En revanche, être célibataire au sens de létat civil semble entraîner, pour les associations culturelles et éducatives, ainsi que pour les associations sportives généralistes, une meilleure visibilité. Lâge et la position dans le cycle de vie induisent donc des effets contrastés. L'engagement associatif et électoral culmine pour les plus âgées mais ne pas être en couple et être jeune peut parfois aller de pair avec certaines formes de participation à l'espace public, comme le sport en club.

\section{L' "ancrage" en Espagne, un effet positif sur la visibilité}

On envisage souvent la durée de l'étape migratoire comme un vecteur positif d'installation dans le pays d'accueil. Ici, nous disposons de davantage de variables permettant de parler d'un ancrage en Espagne : l'année d'arrivée, la possession de la nationalité espagnole, les projets migratoires individuels et familiaux, l'investissement dans un bien immobilier en Espagne ${ }^{18}$. D'une manière générale, ce sont les femmes les plus anciennement installées 
en Espagne qui se retrouvent surreprésentées dans les ONG, les associations culturelles et éducatives et parmi les votantes aux élections municipales. Disposer de la nationalité espagnole semble également faciliter la participation aux ONG, aux associations culturelles et bien sûr aux élections municipales. Les migrantes ayant le projet de migrer vers un autre pays dans les cinq prochaines années sont systématiquement sousreprésentées dans les associations et lors des élections. De même, ne pas envisager de regroupement familial en Espagne se conjugue souvent avec une faible participation associative ou électorale.

L'investissement économique ou immobilier en Espagne a, en revanche, des effets contrastés sur la participation, par exemple, sur la sousreprésentation dans les associations culturelles. On retiendra cependant une surreprésentation des "investisseuses" lors des élections municipales et dans les associations généralistes.

D’une manière générale, être ancré en Espagne semble donc jouer positivement sur la participation associative et électorale.

\section{Un effet de la position sociale à nuancer}

Comme nous l'avons observé plus haut, appréhender la position sociale des migrantes est peu aisé en raison des asymétries entre diplômes et niveau de qualification de l'emploi. Il faut remarquer la sous-représentation généralisée des migrantes n'ayant pas fait d'études ou seulement des études primaires, allant de pair avec la surreprésentation généralisée des migrantes ayant fait des études supérieures. Parallèlement, si on s'intéresse à la profession exercée en Espagne, les professions non qualifiées et les employées de services sont sousreprésentées, tandis que les cadres sont quasi systématiquement surreprésentées.

\section{/// Figure 2 : Participation associative des migrants et des migrantes selon le type d'association en Espagne en 2007.}

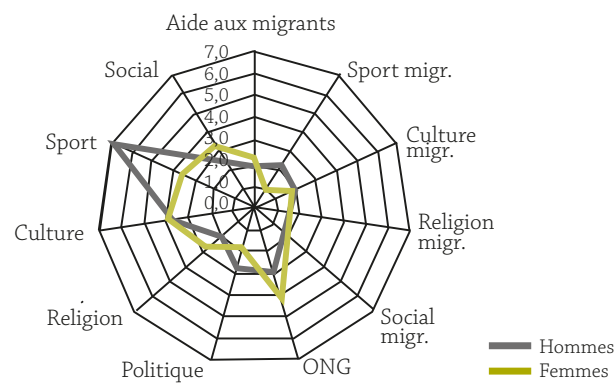

Source : Instituto Nacional de Estadísticas (Espagne), op cit. Lecture : En 2007, en Espagne, 7 \% des hommes migrants participaient à une association sportive contre seulement 3,5\% des femmes migrantes. 2,4\% des hommes participaient à des associations sportives destinées spécifiquement aux migrants.

Ne concluons pas pour autant à une invisibilité des femmes les moins qualifiées dans l'espace public. Par exemple, les femmes ne sachant ni lire ni écrire ont autant de "chances" de participer à une association éducative ou culturelle destinée aux migrants que les femmes sachant lire et écrire. On peut faire l'hypothèse que ces dernières sont particulièrement présentes dans des associations consacrées à des cours d'alphabétisation. De même, être en recherche d'emploi ne s'accompagne pas nécessairement d'une invisibilité dans les associations. Les femmes "au foyer", c'est-à-dire ayant des charges de travail dans leur domicile, ont également plus de chances de participer à des associations culturelles et éducatives pour migrants que les autres. La maitrise de la langue du pays accueil, quant à elle, induit des effets contrastés sur la participation à l'espace public. D'une part, on constate une sousreprésentation des femmes dont l'espagnol est la langue maternelle, sauf dans les élections municipales et dans les associations généralistes à vocation éducative ou culturelle. D’autre part, pour les non- 
hispanophones d'origine, la bonne maitrise de l'espagnol ne s'accompagne pas systématiquement d'une meilleure représentation dans les associations : les migrantes ne parlant pas du tout l'espagnol sont bien représentées dans les associations d'aide aux migrantes, dans les associations culturelles et éducatives (y compris généralistes) et dans les ONG. Au-delà de la position sociale des individus, le fait de résider dans des grandes régions métropolitaines (Madrid, Catalogne et Valence) semble jouer positivement sur la visibilité dans les associations culturelles ou éducatives, phénomène que l'on peut interpréter comme le produit d'une répartition inégale de l'offre associative entre les grandes métropoles et les plus petites villes.

\section{Conlusion}

Cet article permet d'entrevoir, à titre d'étude exploratoire, les caractéristiques de la participation aux associations et aux élections municipales des migrantes en Espagne. L'hypothèse selon laquelle les femmes migrantes seraient moins visibles que les hommes migrants ne tient pas vraiment. Les migrantes sont en réalité visibles dans d'autres recoins de l'espace public (ONG, associations d'aide aux migrants, associations à vocation sociale, organisations religieuses) que les migrants (organisations sportives et politiques). L'interrogation de la participation des femmes migrantes à l'espace public en Espagne se heurte, cependant, à la diversité des situations des migrantes. Il convient ainsi de distinguer différentes modalités de visibilité des migrantes dans l'espace public. Une approche par le pays d'origine a permis de montrer qu'en 2007, certains groupes nationaux (les Britanniques, les Uruguayennes, les Argentines, les Mexicaines, les Vénézuéliennes, les Allemandes, les Suisses et les Françaises) participaient plus que les autres (les Bulgares, les Roumaines, les Polonaises, les Russes, les Équatoriennes, les Boliviennes, les Dominicaines, les Colombiennes et les
Marocaines) à l'espace public. Cependant, derrière ces inégalités selon le pays d'origine se cachent des effets de structure. Une analyse des caractéristiques socio-démographiques a montré que les femmes les plus jeunes et les moins "installées" en Espagne avaient peu de chances d'être visibles dans l'espace public. En revanche, l'effet de la position sociale est à nuancer. S'il est avéré que les plus qualifiées se déplacent plus souvent aux bureaux de vote, lorsqu'elles en ont le droit, et qu'elles participent plus souvent aux organisations politiques et syndicales, notre analyse a montré que certaines femmes peu qualifiées, notamment celles qui ne maîtrisent pas du tout l'espagnol, sont surreprésentées dans les associations à vocation culturelle ou éducative.

Depuis 2007, période de collecte des données de l'ENI, l'espace public espagnol a bien changé. La crise économique et financière s'est traduite en crise politique, les partis traditionnels laissant le champ politique libre aux nouveaux mouvements sociaux. Du mouvement des Indignés 15-M en 2011 aux percées électorales de Podemos en 2015, la participation à l'espace public semble sêtre démocratisée en Espagne. Dans ce contexte, on peut faire l'hypothèse que la visibilité dans l'espace public ne prend plus forcément la forme d'une participation officielle, ni d'un encartement dans un parti - critères de visibilité selon la définition de notre article -, mais une forme plus souple, par exemple une participation épisodique à des rassemblements réguliers comme lors des manifestions des Indignés de 2011. La position des femmes migrantes dans l'espace public s'est sans doute renforcée par ce biais, mais également grâce aux réformes du droit de vote des étrangers extracommunautaires aux élections municipales (désormais les Colombiennes, les Équatoriennes ou encore les Boliviennes peuvent voter), à la féminisation des flux migratoires vers l'Espagne, au retour vers le pays d'origine des hommes migrants dont le secteur d'emploi dominant - le bâtiment - a particulièrement été affecté par la crise. 